Revista Internacional de Ciencias Podológicas

ISSN: 1989-5151

http://dx.doi.org/10.5209/RICP.59825

\title{
Fracturas de estrés en atletas
}

Grande del Arco, Jessica ${ }^{1}$

Fecha de recepción: 2 de diciembre de 2017 / Fecha de aceptación: 2 de febrero de 2018

Resumen. La fractura por estrés se trata de una solución de continuidad parcial o completa de un hueso como resultado de cargas excesivas o repetidas, a una determinada intensidad máxima, resultando en una mayor reabsorción frente a una insuficiente formación de tejido óseo. La distribución de las fracturas de estrés más comunes es en tibia, huesos del tarso y metatarsianos y varios son los factores de riesgo para producirse dicha lesión como los factores extrínsecos relacionados con equipamiento deportivo y acondicionamiento físico y Los factores intrínsecos como son la edad, el sexo, la etnicidad, la densidad ósea y a nivel metabólico. Este articulo tiene como objetivo realizar una revisión sistemática sobre los huesos de miembros inferiores más sensibles de producir dicha lesión, la distribución de la edad de los pacientes atletas, el sexo, los factores de riesgo de tipo intrínseco como extrínsecos, el tratamiento a aplicar y las recomendaciones que debemos comunicar como profesionales sanitarios para la prevención de la lesión en el entrenamiento.

\begin{abstract}
Stress fracture is a solution of partial or complete continuity as a result of excessive or repeated loads, an acute and severe tide, resulting in a greater resorption compared to a weak tissue formation. The distribution of the most common stress fractures in the tibia, the tarsal bones and the metatarsals and several are the risk factors for the production of the injury such as extrinsic factors related to sports equipment and physical conditioning and factors intrinsic factors such as age, sex, race, bone density and metabolic level. This article aims to perform a systematic review on the bones of the smallest, most sensitive members of the lesion, the distribution of patients' age, sex, intrinsic and extrinsic risk factors, the treatment to be applied the recommendations that should be communicated as health services for the prevention of injury in training
\end{abstract}

Keywords: Stress Fracture And Athletes; Stress fracture and lower limb; stress fracture in foot

Los autores declaran no tener ningún tipo de interés económico o comercial.

Sumario: 1. Introducción. 2. Material y métodos. 3. Estrategias de búsqueda. 4. Resultados. 4.1. Localización de las fracturas de estrés. 4.2. Distribución estadística en función del sexo del sujeto. 4.3. Distribucón según la edad. 4.4. Factores de riesgo. 4.5. Tratamiento. 4.6. Prevención de la lesión. 5. Discusión. 6. Conclusión. 7. Bibliografía. 8. Anexo.

Cómo citar: Grande del Arco, J. (2018) Fracturas de estrés en atletas, en Revista internacional de ciencias podológicas $12(2), 63-71$

\footnotetext{
Grado en Podología. Universidad Complutense de Madrid Máster universitario en Investigación en Podología Universidad Rey Juan Carlos jessicagrandedelarco@gmail.com Calle Goya 57,1Derecha, 28001
} 


\section{Introducción}

Las fracturas por estrés fueron descritos por primera vez en 1855 por Breihaupt $^{1}$ entre los soldados que presentaban dolor plantar y edema después de largas marchas ${ }^{2}$. Los atletas y el cuerpo militar representa aproximadamente el $10 \%$ de todas las lesiones ortopédicas ${ }^{3}$. Para los atletas, la primera descripción clínica fue dada por Devas en $1958^{4}$, basado únicamente en los resultados de las lesiones de estrés en radiografías simples.

La fractura por estrés se define como una solución de continuidad parcial o completa de un hueso como resultado de cargas excesivas o repetidas, a una intensidad máxima, resultando en una mayor reabsorción frente a una insuficiente formación de tejido óseo ${ }^{2}$.

Aunque las fracturas de estrés pueden afectar a todos los tipos de tejido óseo, son más comunes en los huesos que soportan peso corporal como los miembros inferiores (tibia, $49 \%$; huesos del tarso, 25\%; metatarsianos, $9 \%)^{3}$. Los estudios sobre los corredores revelan una mayor incidencia de fracturas por estrés en la tibia, seguido de los metatarsianos, el peroné, fémur y el hueso navicular ${ }^{5,6}$.

Los corredores pueden desarrollar una fractura por estrés del maléolo medial, el extremo distal del peroné, calcáneo, en metatarsianos, y en el sesamoideo medial ${ }^{8-10}$.

Desde un punto de vista biomecánico, las fracturas por fatiga son el resultado de la acción muscular específica, cíclica y repetitiva hasta el agotamiento, con la transferencia de carga al hueso superior a su capacidad de adaptación ${ }^{10,11}$. Como resultado, la mayoría de las fracturas por estrés se encuentran en las áreas que sufren estrés por cizallamiento $^{10}$.

La sospecha de fractura por estrés se basa en los datos de la historia clínica, el examen físico general y ortopédico9 ${ }^{9}$. Es importante establecer la relación entre el inicio de los síntomas dolorosos y la actividad física, por lo general se realiza de forma repetitiva, cambios bruscos en la cantidad de entrenamiento y la presencia de factores de riesgo $0^{10,12,13}$.

\section{Material y métodos}

Este trabajo tiene como objetivo, sintetizar la evidencia disponible sobre las lesiones por fracturas de estrés en atletas. Para ello utilizaremos unos metaanálisis y revisiones sistemáticas sobre el tema.

Esta cuestión fue separada en tres términos diferentes de búsqueda y 8 bases de datos electrónicos (MEDLINE, CINAHL, PUBMED, WEB OF SCIENCE, SCOPUS, EBSCOHOST Y COCHRANE LIBRARY) (Tabla 1). Debido a que encontramos múltiples artículos donde mencionan las lesiones producidas en pie y tobillo, formas de abordar la patología, métodos de diagnóstico, pero nos centraros en las lesiones producidas en el miembro inferior

\section{Estrategias de búsqueda}

Se revisaron de manera independiente todos los estudios recuperados siguiendo los criterios de búsqueda (Tabla 1). Se obtuvo los artículos completos cuando el resumen resultaba dudoso. Se revisó la lista de referencias de cada uno de los artículos, incluyendo exclusivamente revisiones sistemáticas y meta-análisis.

Encontramos quinientos setenta y nueve referentes a las fracturas de estrés en atletas, sobre las fracturas en el miembro inferior, todos están redactados exclusivamente en inglés. Son excluidos quinientos sesenta y seis tras la revisión de resúmenes, seleccionando en principio los que hacen referencia a los factores de riesgo y tratamientos actuales (Figura 1), que cumplen con los criterios de inclusión y exclusión (Tabla 2), uno de ellos es un meta-análisis, el resto son revisiones sistemáticas.

\section{Resultados}

\subsection{Localización de las fracturas de estrés}

En el estudio de Mckenzie y colaboradores ${ }^{3}$ presentaron 320 casos de los cuales 145 de los sujetos eran masculinos y 157 femeninos y se realizó un estudio de distribución en función del sexo y la localización de la lesión. Mostramos en la figura 2 en la cual la tibia ocupa el puesto del hueso más frecuente en desarrollar una fractura por estrés $49.1 \%$ y los huesos tarsales secundariamente con $25.3 \%$. A su vez los huesos metatarsales presentan un $8.8 \%$, fémur un $7.2 \%$, peroné $6,6 \%$ y pelvis $1,6 \%$, sesamoideos $0.9 \%$ y $0.6 \%{ }^{3}$. 
En el lado derecho tenemos un porcentaje de $38.1 \%(\mathrm{~N}=122)$ y en el lado izquierdo $45.3 \%(\mathrm{~N}=145)$. Los casos de fractura por estrés de manera bilateral lo encontramos en $16.6 \%$ de los casos $(\mathrm{N}=53)^{3}$ (Fig 1).

\subsection{Distribución estadística en función del sexo del sujeto}

En la revisión de Bennel y colaboradores muestra los promedios ( $\pm \mathrm{DE}$ ) para el contenido total de densidad ósea regional comparado entre atletas con y sin fracturas por estrés ${ }^{15}$. Las mujeres atletas que desarrollaron fracturas de estrés tenían un valor significativamente menor de contenido mineral óseo de la zona lumbar,espina dorsal y del $\mathrm{pie}^{15}$. No hubo diferencias significativas de masa ósea entre los atletas masculinos que desarrollaron fracturas por estrés y los que no presentaban dicha afección ${ }^{15}$.

Sin embargo, parecía haber una tendencia de menor masa ósea en el grupo de fractura por estrés masculino con déficits de $6.8 \%$ de contenido total de mineral oseo y $4.0 \%$ para la densidad mineral ósea de tibia y peroné ${ }^{15}$.

Se muestra déficits $( \pm \mathrm{SE})$ en la masa ósea de los atletas con fracturas por estrés como un porcentaje de aquellos sin ellas ${ }^{15}$.

En el subgrupo de atletas que sufrieron fracturas de estrés tibial, se comparó la densidad mineral ósea tibial y fibular en el lado lesionado con el promedio tibial y densidad mineral del peroné en atletas sin fracturas de estrés ${ }^{15}$.

El grupo de fractura por estrés tibial femenino tenía una densidad ósea tibial y fibular de $8,1 \%$ que el grupo de atletas femeninos sin fractura sin estrés $\left(\mathrm{P}<0.01^{15}\right.$. En grupo de fractura por estrés masculino tenía una densidad tibial menor tibial y fibular de $4,0 \%$ menor que el grupo sin fracturas de estrés aunque este hallazgo no fue significativo $(\mathrm{P}=0.17)^{15}$.

Cuando los valores de masa ósea para las atletas con las fracturas por estrés se compararon con las de un paciente grupo de mujeres no atletas $(\mathrm{N}=28)$, los atletas con las lesiones óseas tenían significativamente más miembros inferiores densidad mineral ósea $(\mathrm{P}<0.05)$ y cuerpo total similar contenido mineral óseo y hueso de la espina lumbar mineral densidad ${ }^{15}$.

No hubo diferencia entre los nervios femeninos o masculinos ${ }^{15}$.
Las atletas femeninas son más propensos a las fracturas por estrés en desarrollo ${ }^{16}$. El aumento creciente de esta patología entre los atletas femeninos se relaciona con factores que caracterizan triada atlética femenina: trastornos de la alimentación, trastornos menstruales y la densidad ósea baja ${ }^{6,9,10}$ mayor prevalencia de trastornos (tales como bulimia, anorexia nerviosa, ingesta de laxantes y diuréticos) que come se ha encontrado entre los atletas femeninos ${ }^{17}$. Las irregularidades en el ciclo menstrual (hipoestrogenismo) se correlacionan con la pérdida ósea temprana, la reducción de la mineralización del osteoide y, en consecuencia, la prevalencia de las fracturas por estrés en mujeres ${ }^{18}$.

\subsection{Distribucón según la edad}

En el estudio mencionado en el anterior apartado, la media fue de 26.7 (13-61 años), con una media en varones de 29.2 años y en mujeres de 25.1 años $^{2}$. El resultado del análisis muestra como las fracturas tarsales y femorales son más comunes en los atletas más adultos y las fracturas de estrés en tibia y peroné en los más jóvenes. Los hallazgos fueron más significativos en la población más adulta de sexo masculino que del femenino $(\mathrm{P}=0.0005)$.

\subsection{Factores de riesgo}

Varios factores contribuyen a la patogénesis de la enfermedad, que se pueden clasificar en 2 sub-tipos: intrínseca y extrínseca. En general, los factores extrínsecos están relacionados con el tipo y ritmo de formación, el uso de calzado inadecuado y equipamiento deportivo, acondicionamiento físico precario, el lugar de entrenamiento, la temperatura del medio ambiente y el tiempo de recuperación insuficiente de las lesiones anteriores. Los factores intrínsecos incluyen la edad, el sexo, la raza, la densidad ósea y la estructura, hormonal, menstrual, metabólicas y el equilibrio nutricional, patrón de sueño y las enfermedades del colágeno ${ }^{9,10,16,20 .}$

Los estudios prospectivos y retrospectivos muestran una mayor incidencia entre los caucásicos en comparación con los sujetos negros e hispanos estadounidenses, los individuos blancos son más susceptibles a las fracturas por estrés ${ }^{18}$. Lo mismo ocurre con la edad: los individuos de mayor edad presentan 
una mayor incidencia de este tipo de fracturas 9 Las fracturas por estrés son menos comunes en niños que en adolescentes y adultos ${ }^{5}$. En relación con el sexo, algunos estudios han demostrado que las mujeres tienen una incidencia de 5 a 10 veces mayor que los hombres ${ }^{9}$.

Con respecto a los factores genéticos, los estudios sobre reclutas militares gemelos idénticos sometidos al mismo tratamiento en cantidad, duración e intensidad revelan fracturas por fatiga en los huesos del metatarso en ambos 9 .

En relación con los factores biomecánicos, un alto arco longitudinal del pie, diferencia en la longitud de las extremidades inferiores y un pie varo marcado asociado con múltiples fracturas de estrés se han observado ${ }^{21-23}$.

Los pies cavo-varos han sido recientemente marcados como un factor de riesgo significativo para las diversas condiciones de sobrecarga, especialmente las fracturas por estrés. Esta forma del pie es conocida por ser relativamente rígida, con una capacidad débil para la atenuación de choque ${ }^{24,25}$. La supinación y pronación del pie están asociados con un aumento significativo en el riesgo de lesiones por estrés ${ }^{26}$.

\subsection{Tratamiento}

El tratamiento de las fracturas por estrés varía de acuerdo con algunas de las características de la fractura, como la ubicación, tipo y tiempo de evolución ${ }^{9}$.

Un plan general se puede establecer divide en dos fases: fase I, o resto modificado, se caracteriza por el control del dolor mediante el uso de fármacos antiinflamatorios, los métodos de fisioterapia para la analgesia y cinesiterapia, la carga de peso-permitido en las actividades diarias y el mantenimiento de aeróbica aptitud sin causar respuestas de estrés anormales en el segmento afectado. Las actividades tales como montar en bicicleta, nadar o correr en el agua son alternativas para mantener la condición física del atleta9.

Fase II comienza desde el momento en que el atleta ya no presenta quejas de dolor, que ocurre generalmente dentro de 10 a 14 días desde el inicio de los síntomas. Se permite un retorno gradual a la deporte basado en la corrección de los factores intrínsecos y extrínsecos ${ }^{9}$.
La mayoría de las fracturas por estrés pueden ser tratados de forma conservadora. Esto implica la inmovilización en una bota, hasta que los síntomas hayan desaparecido, por lo general alrededor de 6 a 8 semanas $^{16}$. Es necesario que las actividades de impacto se eviten pero los entrenamientos de bajo impacto como la natación, el ciclismo y máquinas elípticas se pueden continuar para mantener la capacidad aeróbica ${ }^{22}$. Los exámenes físicos frecuentes son útiles para identificar la resolución de los síntomas. Consideraciones nutricionales son importantes deficiencias en la dieta pueden contribuir al desarrollo de las fracturas por estrés. Los datos recientes recomienda tratamiento quirúrgico precoz de las fracturas con un alto riesgo de estrés a los atletas de élite, debido al alto riesgo de dislocación y no consolidación ${ }^{22,27-29}$.

La estimulación eléctrica también se ha utilizado para el tratamiento de fracturas de estrés con resultados satisfactorios ${ }^{30}$.

\subsection{Prevención de la lesión}

El profesional de la salud es responsable de conocer bien su atleta, buscando detectar factores intrínsecos y extrínsecos concurrentes de los daños causados por los microtraumatismos de repetición, y los de corrección $^{12}$.

La prevención de las lesiones y el pronóstico son de particular importancia para los atletas de competición ya que el objetivo no es solo para empezar a participar de nuevo, pero para competir a un alto nivel, la prevención de consecuencias a largo plazo $^{31}$.

Las estrategias y programas de prevención de lesiones son una parte vital de la educación y la formación de los atletas en todos los niveles $^{31}$. Es importante educar a los atletas que el dolor continuo que dura 3 semanas es una señal de advertencia para el cuerpo, y que el diagnóstico precoz conduce a una recuperación más rápida ${ }^{31,32}$.

Los cambios en el calzado y la superficie para la práctica de la formación pueden ayudar a reducir el número y la gravedad de las lesiones en relación con los pies y los tobillos de los atletas ${ }^{17}$.

El tipo Calzado usado puede tener un papel fundamental en el aumento de las tasas de lesiones. El uso de zapatos ligeros y flexibles con menos apoyo de la parte media del pie pueden producir un riesgo 
para el atleta en riesgo, ya que estos pueden ofrecer menos protección contra las fuerzas potencialmente perjudiciales en el $\mathrm{pie}^{33}$. Una revisión 1999 declaró que el uso de piezas de absorción de choques en el calzado probablemente reduce la incidencia de fracturas por estrés en el personal militar ${ }^{34}$. Otra revisión Cochrane encontró evidencia de que las ortesis adaptadas eran eficaces en el tratamiento de dolor en el pie cavo ${ }^{33}$. Los zapatos con plantillas neutras correr han demostrado recientemente una reducción estadísticamente significativa en la presión plantar en los atletas con pies cavos ${ }^{35}$.

En relación con la nueva fractura, es bien sabido que el regreso al deporte temprana es un riesgo importante, por lo tanto, los atletas deben ser advertidos acerca de la complicación 36-38. En los deportistas de alto nivel, la tomografía computarizada o resonancia magnética deben ser considerados antes de regresar al entrenamiento con el fin de evitar recidiva $^{36}$.

\section{Discusión}

Esta revisión sistemática tiene como objetivo examinar la prevalencia de las fracturas de estrés en atletas en función de la edad y el sexo, además de evidenciar qué huesos del miembro inferior son más sensibles a presentar este tipo de fracturas y los factores de riesgo que la generan.

También se resume los tratamientos más nombrados en las revisiones y los metaanálisis utilizados.

La edad es un factor fundamental debido a que los atletas adultos son más propensos a padecer dicha patología que los adolescentes ${ }^{7}$.

Las fracturas por estrés del pie y el tobillo en deportistas son relativamente poco comunes en el $1 \%$ de todas las lesiones deportivas ${ }^{10}$. Sin embargo, la aplicación de un entrenamiento más riguroso ha contribuido a un aumento de la incidencia de fracturas por estrés ${ }^{39}$. Además, en la población femenina de atletismo es recomendable una exhaustiva revisión debido a los factores de riesgo tales como la densidad ósea y la estructura, hormonal, menstrual, metabólicas y el equilibrio nutricional, patrón de sueño y las enfermedades del colágeno ${ }^{10,40}$.

La tibia ocupa el puesto del hueso más frecuente en desarrollar una fractura por estrés $49.1 \%$ y los huesos tarsales secundariamente con $25.3 \%$. A su vez los huesos metatarsales presentan un $8.8 \%$, fémur un $7.2 \%$, peroné $6,6 \%$ y pelvis $1,6 \%$, sesamoideos medial $0.9 \%$ y lateral $0.6 \%{ }^{3}$.

Las fracturas por estrés del maléolo medial son poco frecuentes y generalmente se encuentran en los atletas que participan en carreras, saltos, patadas y los deportes, aunque también se han implicado fuerzas anormales en el tobillo debido a la tibia y el astrágalo osteofitos $^{22,38}$.

Las fracturas de estrés del astrágalo fueron descritos por primera vez en 1965 por McGlone ${ }^{41}$. Es una lesión relativamente rara; atletas y reclutas militares que realizan actividades de carga axial repetitivos son más propensos a esta lesión ${ }^{10}$.

Las Fracturas por estrés navicular son actualmente considerados de alto riesgo debido a la tasa de pseudoartrosis ${ }^{29,42}$. Los pacientes atletas están involucrados en actividades de carreras de velocidad o de salto explosivos y se quejan de dolor en el dorso de la parte media del pie o a lo largo del arco longitudinal medial de actividad ${ }^{4}$.

Estas fracturas por estrés se producen con mayor frecuencia en el segundo y tercer metatarsianos y son relativamente comunes $10,42,43$. Se consideran de riesgo alto o bajo, dependiendo de la actividad y la ubicación ${ }^{10}$, 42,43 .

La presentación típica de las fracturas de estrés quinto metatarsiano se diferencia de las fracturas agudas clínica y radiográficamente. El dolor ha sido por lo general presente durante varias semanas, y la fractura es clásicamente ubicado en el cruce diafisaria-metafisaria ${ }^{44}$. La fuerza de aducción repetitivo con el tobillo en flexión plantar a menudo hace que estas fracturas por estrés debido a la atracción de la fascia plantar. Como tales, son vistos con frecuencia en los jugadores de baloncesto ${ }^{10,42,}$ 43.

El sesamoideo medial se lesiona más comúnmente debido a su posición directamente debajo de la cabeza del primer metatarsiano ${ }^{10,}$ 42,43 . Las actividades relacionadas con la dorsiflexión repetitiva, por la fuerza de los dedos de los pies están asociados con mayor frecuencia ${ }^{45}$.

El hinchazón o incluso saltones tejidos blandos pueden estar presentes, y el dolor se reproduce con la dorsiflexión forzada ${ }^{45}$.

La incidencia de fracturas por estrés del calcáneo es más alta en los reclutas militares y 
corredores de larga distancia ${ }^{10,42,43,46}$. A prueba de compresión del calcáneo positiva con una cierta cantidad de hinchazón es generalmente presente $^{10,42,43,46}$.

Las fracturas de estrés cuneiformes fueron reportados por primera vez en 1936 como una fractura marcha de la cuña medial también se han reportado fracturas por sobrecarga de la cuña media y lateral ${ }^{47}$ huesos a diferencia de una diáfisis, la cuña y el cuboides no pueden mostrar el callo perióstico usual ${ }^{47}$.

\section{Conclusión}

Las fracturas de estrés representan el 10\% de las lesiones generadas en atletas. La tibia es uno de los huesos más frecuentes en presentar dicha patología, pero también los huesos del pie forman un gran porcentaje.
Los factores de riesgo pueden dividirse en dos grupos intrínsecos como es la densidad ósea y la estructura, hormonal, menstrual, metabólicas y el equilibrio nutricional, patrón de sueño y las enfermedades del colágeno, la edad y extrínsecos como puede ser la temperatura del medio ambiente y el tiempo de recuperación insuficiente de las lesiones anteriores, el calzado y el terreno.

Las atletas femeninas están expuestas a mayor riesgo debido a los cambios hormonales y a la menor densidad ósea del tejido. En cuanto a la edad, los atletas adultos presentan mayor probabilidad de lesión.

Para ayudar a prevenir la lesión debemos informar al paciente que el diagnóstico precoz conduce a una recuperación más rápida además de los cambios de terreno y calzado pueden ayudar a reducir el número y la gravedad de las lesiones en relación con los pies y los tobillos de los atletas.

\section{Bibliografía}

1. Breithaupt J. Zur pathologie des menschlichen fussess. Medizin Zeitung. 1855;24:169-77.

2. Fitch KD. Stress fractures of the lower limbs in runners. Aust Fam Physician. 1984;

3. Matheson GO, Clement DB, McKenzie DC, Taunton JE, Lloyd-Smith DR, MacIntyre JG. Stress fractures in athletes. A study of 320 cases. Am J Sports Med. 1971;

4. DEVAS MB. Stress fractures of the tibia in athletes or shin soreness. J Bone Joint Surg Br [Internet]. 1958 May [cited 2017 Dec 11];40-B(2):227-39. Available from: http://www.ncbi.nlm.nih.gov/ pubmed/13539106

5. Bennell KL, Brukner PD. Epidemiology and site specificity of stress fractures. Clinics in Sports Medicine. 1997.

6. e Côrte ACR e., Hernandez AJ. Termografia médica infravermelha aplicada à medicina do esporte. Rev Bras Med do Esporte. 2016;

7. Reeder MT, Dick BH, Atkins JK, Pribis AB, Martinez JM. Stress fractures. Current concepts of diagnosis and treatment. Sports Med. 1996;

8. Haverstock BD. Foot and Ankle Imaging in the Athlete. Clinics in Podiatric Medicine and Surgery. 2008.

9. Bennell KL, Malcolm SA, Thomas SA, Reid SJ, Brukner PD, Ebeling PR, et al. Risk factors for stress fractures in track and field athletes. A twelve-month prospective study. Am J Sport Med. 1996;

10. Boden BP, Osbahr DC. High-risk stress fractures: evaluation and treatment. J Am Acad Orthop Surg. 2000;

11. Sonoda N, Chosa E, Totoribe K, Tajima N. Biomechanical analysis for stress fractures of the anterior middle third of the tibia in athletes: nonlinear analysis using a three-dimensional finite element method. J Orthop Sci. 2003;

12. Verma RB, Sherman O. Athletic stress fractures: part I. History, epidemiology, physiology, risk factors, radiography, diagnosis, and treatment. Am J Orthop (Belle Mead NJ). 2001;

13. Knapp TP, Garrett Jr. WE. Stress fractures: general concepts. Clin Sport Med. 1997;

14. Huang X, Lin J, Demner-Fushman D. Evaluation of PICO as a knowledge representation for clinical questions. AMIA Annu Symp Proc. 2006;359-63.

15. Bennell KL, Malcolm SA, Thomas SA, Reid SJ, Brukner PD, Ebeling PR, et al. Risk Factors for Stress Fractures in Track and Field Athletes. Am J Sports Med [Internet]. 1996 Nov 23 [cited 2017 Nov 15];24(6):810-8. Available from: http://journals.sagepub.com/doi/10.1177/036354659602400617

16. Korpelainen R, Orava S, Karpakka J, Siira P, Hulkko A. Risk factors for recurrent stress fractures in athletes. Am J Sport Med. 2001; 
17. Barrow GW, Saha S. Menstrual irregularity and stress fractures in collegiate female distance runners. Am J Sports Med [Internet]. 1988 May 23 [cited 2017 Nov 15];16(3):209-16. Available from: http:// journals.sagepub.com/doi/10.1177/036354658801600302

18. Monteleone GP. Stress fractures in the athlete. Orthop Clin North Am [Internet]. 1995 Jul [cited 2017 Nov 23];26(3):423-32. Available from: http://www.ncbi.nlm.nih.gov/pubmed/7609957

19. Matheson GO, Clement DB, Mckenzie DC, Taunton JE, Lloyd-Smith DR, Macintyre JG. Stress fractures in athletes. Am J Sports Med [Internet]. 1987 Jan 23 [cited 2017 Nov 15];15(1):46-58. Available from: http://journals.sagepub.com/doi/10.1177/036354658701500107

20. Blivin SJ, Martire JR, McFarland EG. Bilateral midfibular stress fractures in a collegiate football player. Clin J Sport Med [Internet]. 1999 Apr [cited 2017 Nov 23];9(2):95-7. Available from: http://www.ncbi. nlm.nih.gov/pubmed/10442625

21. Giladi M, Milgrom C, Simkin A, Danon Y. Stress fractures. Am J Sports Med [Internet]. 1991 Nov 23 [cited 2017 Nov 15];19(6):647-52. Available from: http://journals.sagepub.com/ doi/10.1177/036354659101900617

22. Kor A, Saltzman AT, Wempe PD. Medial malleolar stress fractures. Literature review, diagnosis, and treatment. J Am Podiatr Med Assoc [Internet]. [cited 2017 Nov 23];93(4):292-7. Available from: http:// www.ncbi.nlm.nih.gov/pubmed/12869598

23. Popovic N, Jalali A, Georis P, Gillet P. Proximal fifth metatarsal diaphyseal stress fracture in football players. Foot Ankle Surg. 2005;

24. Pearce CJ, Brooks JH, Kemp SP, Calder JD. The epidemiology of foot injuries in professional rugby union players. Foot Ankle Surg. 2011;

25. Raikin SM, Slenker N, Ratigan B. The Association of a Varus Hindfoot and Fracture of the Fifth Metatarsal Metaphyseal-Diaphyseal Junction. Am J Sports Med. 2008;

26. Cain LE, Nicholson LL, Adams RD, Burns J. Foot morphology and foot/ankle injury in indoor football. J Sci Med Sport. 2007;

27. Palamarchuk HJ, Sabo M. Fibular stress fracture in a female runner. A case report. J Am Podiatr Med Assoc [Internet]. 1998 Jan [cited 2017 Nov 23];88(1):34-6. Available from: http://www.japmaonline. org/doi/abs/10.7547/87507315-88-1-34

28. Hootman JM, Dick R, Agel J. Epidemiology of collegiate injuries for 15 sports: Summary and recommendations for injury prevention initiatives. Journal of Athletic Training. 2007.

29. Brockwell J, Yeung Y, Griffith JF. Stress fractures of the foot and ankle. Sport Med Arthrosc. 2009;

30. Benazzo F, Mosconi M, Beccarisi G, Galli U. Use of capacitive coupled electric fields in stress fractures in athletes. Clin Orthop Relat Res. 1995;

31. Ohta-Fukushima M, Mutoh Y, Takasugi S, Iwata H, Ishii S. Characteristics of stress fractures in young athletes under 20 years. J Sports Med Phys Fitness. 2002;

32. Schwellnus MP, Jordaan G, Noakes TD. Prevention of common overuse injuries by the use of shock absorbing insoles. Am J Sports Med [Internet]. 1990 Nov 23 [cited 2017 Nov 23];18(6):636-41. Available from: http://www.ncbi.nlm.nih.gov/pubmed/2285093

33. Hawke FE, Burns J, Radford JA, Du Toit V. Custom-made foot orthoses for the treatment of foot pain. Cochrane Database of Systematic Reviews. 2007.

34. Wegener C, Burns J, Penkala S, Sc GDES. Effect of Neutral-Cushioned Running Shoes on Plantar Pressure Loading and Comfort in Athletes with Cavus Feet: A Crossover Randomized Controlled Trial. Am J Sports Med. 2008;

35. Wright RW, Fischer DA, Shively RA, Heidt RS, Nuber GW. Refracture of Proximal Fifth Metatarsal (Jones) Fracture After Intramedullary Screw Fixation in Athletes. Am J Sports Med. 2000;

36. Roche AJ, Calder JDF. Treatment and return to sport following a Jones fracture of the fifth metatarsal: A systematic review. Knee Surgery, Sport Traumatol Arthrosc. 2013;

37. Larson CM, Almekinders LC, Taft TN, Garrett WE. Intramedullary screw fixation of Jones fractures. Analysis of failure. Am J Sport Med. 2002;

38. Sherbondy PS, Sebastianelli WJ. Stress fractures of the medial malleolus and distal fibula. Clin Sports Med [Internet]. 2006 Jan [cited 2017 Nov 23];25(1):129-37, x. Available from: http://linkinghub. elsevier.com/retrieve/pii/S0278591905000724

39. Wasserstein D, Spindler KP. Stress Fractures in Athletes. Stress Fractures in Athletes: Diagnosis and Management. 2015.

40. Maitra RS, Johnson DL. Stress fractures: Clinical history and physical examination. Clinics in Sports Medicine. 1997.

41. McGlone J. Stress fracture of the talus. J Am Podiatr Med Assoc [Internet]. 1965 Dec [cited 2017 Dec 11];55(12):814-7. Available from: http://www.ncbi.nlm.nih.gov/pubmed/5846443

42. Gehrmann RM, Renard RL. Current Concepts Review: Stress Fractures of the Foot. Foot Ankle Int. 2006; 
43. Chuckpaiwong B, Cook C, Nunley JA. Stress Fractures of the Second Metatarsal Base Occur in Nondancers. Clin Orthop Relat Res. 2007;

44. Torg JS, Balduini FC, Zelko RR, Pavlov H, Peff TC, Das M. Fractures of the base of the fifth metatarsal distal to the tuberosity. Classification and guidelines for non-surgical and surgical management. J Bone Joint Surg Am [Internet]. 1984 Feb [cited 2017 Dec 11];66(2):209-14. Available from: http://www.ncbi. nlm.nih.gov/pubmed/6693447

45. Biedert R, Hintermann B. Stress Fractures of the Medial Great Toe Sesamoids in Athletes. Foot Ankle Int [Internet]. 2003 Feb 28 [cited 2017 Dec 11];24(2):137-41. Available from: http://www.ncbi.nlm.nih. gov/pubmed/12627621

46. Sormaala MJ, Niva MH, Kiuru MJ, Mattila VM, Pihlajamäki HK. Stress Injuries of the Calcaneus Detected with Magnetic Resonance Imaging in Military Recruits. J Bone Jt Surg [Internet]. 2006 Oct 1 [cited 2017 Dec 11];88(10):2237. Available from: http://www.ncbi.nlm.nih.gov/pubmed/17015602

47. Meurman KOA. Less common stress fractures in the foot. Br J Radiol [Internet]. 1981 Jan [cited 2017 Dec 11];54(637):1-7. Available from: http://www.ncbi.nlm.nih.gov/pubmed/6108790

\section{Anexo}

Tabla 1. Resultados de las estrategias de búsqueda

\begin{tabular}{|l|l|l|l|l|l|l|l|}
\hline $\begin{array}{c}\text { PALABRAS } \\
\text { CLAVE/ } \\
\text { KEYWORDS }\end{array}$ & MEDLINE & CINAHL & $\begin{array}{c}\text { Web of } \\
\text { Science }\end{array}$ & PubMed & Scopus & $\begin{array}{c}\text { Ebsco } \\
\text { host }\end{array}$ & $\begin{array}{c}\text { Cochrane } \\
\text { library }\end{array}$ \\
\hline $\begin{array}{l}\text { Stress Fracture } \\
\text { And Athletes }\end{array}$ & 0 & 0 & 0 & 24 & 379 & 0 & 8 \\
\hline $\begin{array}{l}\text { Stress fracture } \\
\text { and lower limb, }\end{array}$ & 0 & 0 & 0 & 28 & 21 & 0 & 2 \\
\hline $\begin{array}{l}\text { stress fracture } \\
\text { in foot }\end{array}$ & & & 24 & 86 & & 4 \\
\hline
\end{tabular}

Tabla 2. Criterios de inclusión y exclusión

\begin{tabular}{|l|l|}
\hline CRITERIO DE INCLUSIÓN & CRITERIO DE EXCLUSIÓN \\
\hline - Revisión sistemática & - Artículos no publicados \\
- Meta-análisis & - Revisiones \\
- Con antigüedad de hace 5-10 años de & - Artículos no publicados en ingles \\
publicación & - Ensayos de control aleatorizado \\
- Miembro inferior & - Ensayos clínicos \\
& - Casos clínicos \\
\hline
\end{tabular}




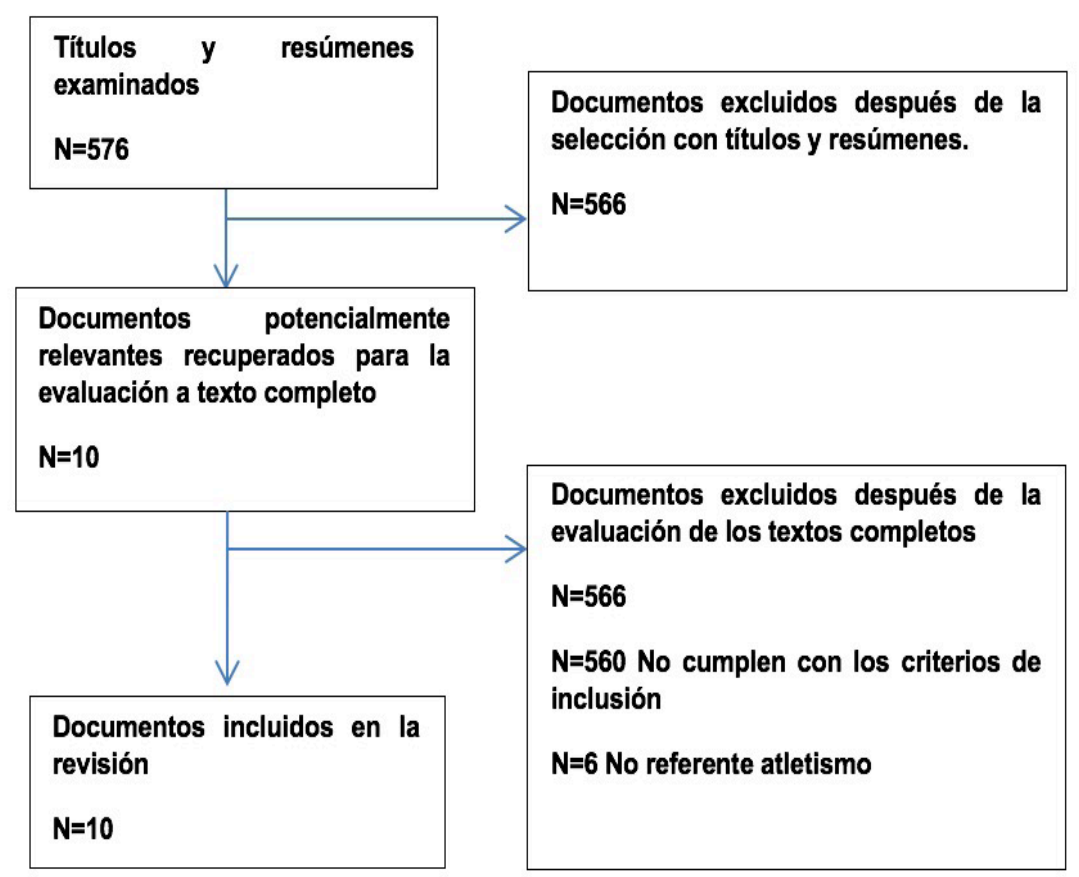

Figura 1. Revisión de la literatura

Porcentaje de Fracturas de estrés (Por localizacion del miembro inferior)

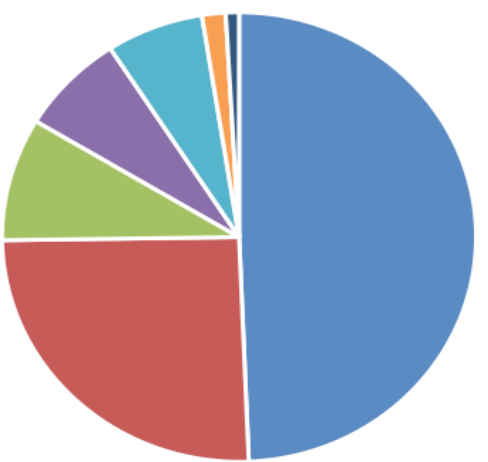

- Tibia - Huesos tarsales " Metatarso - Fémur = Peroné " Pelvis — Sesamoideos

Figura 2: Porcentaje de incidencia de fracturas por estrés en los huesos de los miembros inferiores. 